\title{
Interventions for preventing injuries caused by impaired alertness in individuals with jet lag and shift work disorder (Protocol)
}

\author{
Ker K, Edwards PJ, Roberts I, Blackhall K, Felix LM
}

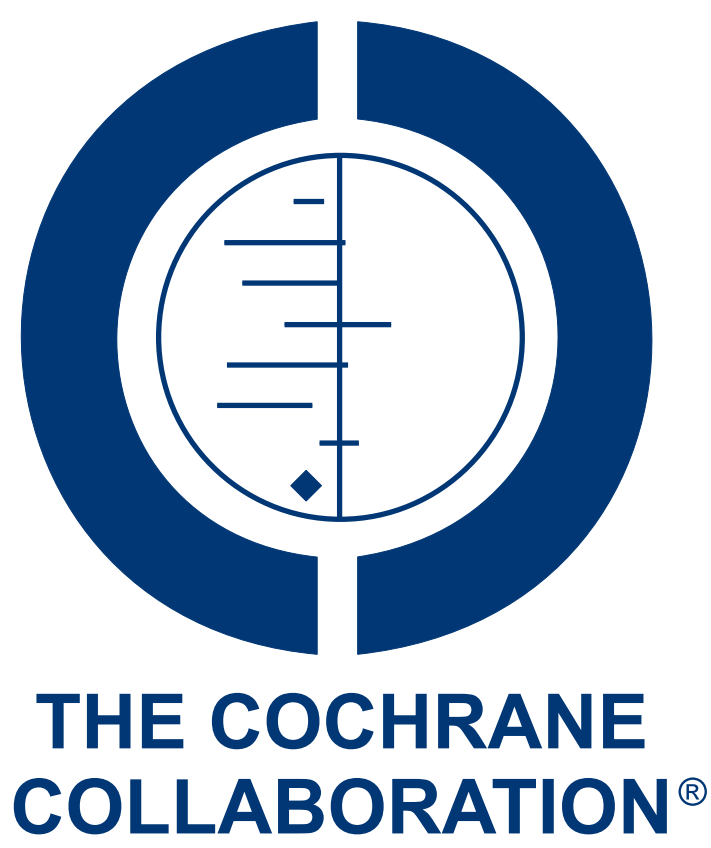

This is a reprint of a Cochrane protocol, prepared and maintained by The Cochrane Collaboration and published in The Cochrane Library 2009, Issue 2

http://www.thecochranelibrary.com

\section{WILEY}

Interventions for preventing injuries caused by impaired alertness in individuals with jet lag and shift work disorder (Protocol) Copyright $\odot 2009$ The Cochrane Collaboration. Published by John Wiley \& Sons, Ltd. 
TABLE OF CONTENTS

HEADER . . . . . . . . . . . . . . . . . . . . . . . . . . . . . . . . . . . . 1

ABSTRACT . . . . . . . . . . . . . . . . . . . . . . . . . . . . . . . . . . . . . . . 1

BACKGROUND . . . . . . . . . . . . . . . . . . . . . . . . . . . . . . . . . . . .

OBJECTIVES . . . . . . . . . . . . . . . . . . . . . . . . . . . . . . . . . . . . . . . .

METHODS . . . . . . . . . . . . . . . . . . . . . . . . . . . . . . . . . . . . . . 44

ACKNOWLEDGEMENTS . . . . . . . . . . . . . . . . . . . . . . . . . . . . . . . . . . . . . . . .

REFERENCES . . . . . . . . . . . . . . . . . . . . . . . . . . . . . . . . . . . . . . 6

HISTORY . . . . . . . . . . . . . . . . . . . . . . . . . . . . . . . . . . . . . . . 8

CONTRIBUTIONS OF AUTHORS . . . . . . . . . . . . . . . . . . . . . . . . . . . . . . . . . . . . . .

DECLARATIONS OF INTEREST . . . . . . . . . . . . . . . . . . . . . . . . . . . . . . . . . . 8

SOURCES OF SUPPORT . . . . . . . . . . . . . . . . . . . . . . . . . . . . . . . . . . . . . . . . . . . . . .

Interventions for preventing injuries caused by impaired alertness in individuals with jet lag and shift work disorder (Protocol)

Copyright $\odot 2009$ The Cochrane Collaboration. Published by John Wiley \& Sons, Ltd. 


\title{
[Intervention Protocol]
}

\section{Interventions for preventing injuries caused by impaired alertness in individuals with jet lag and shift work disorder}

\author{
Katharine Ker ${ }^{1}$, Philip James Edwards ${ }^{2}$, Ian Roberts ${ }^{1}$, Karen Blackhall ${ }^{1}$, Lambert M Felix ${ }^{2}$ \\ ${ }^{1}$ Cochrane Injuries Group, London School of Hygiene \& Tropical Medicine, London, UK. ${ }^{2}$ Department of Epidemiology \& Population \\ Health, London School of Hygiene \& Tropical Medicine, London, UK
}

Contact address: Katharine Ker, Cochrane Injuries Group, London School of Hygiene \& Tropical Medicine, Room 280, North Courtyard, Keppel Street, London, WC1E 7HT, UK. katharine.ker@1shtm.ac.uk.

Editorial group: Cochrane Injuries Group.

Publication status and date: New, published in Issue 2, 2009.

Citation: Ker K, Edwards PJ, Roberts I, Blackhall K, Felix LM. Interventions for preventing injuries caused by impaired alertness in individuals with jet lag and shift work disorder. Cochrane Database of Systematic Reviews 2009, Issue 2. Art. No.: CD007741. DOI: 10.1002/14651858.CD007741.

Copyright (C) 2009 The Cochrane Collaboration. Published by John Wiley \& Sons, Ltd.

\begin{abstract}
A B S T R A C T
This is the protocol for a review and there is no abstract. The objectives are as follows:

To assess the effects of interventions for preventing injuries caused by impaired alertness in persons with jet lag or shift work disorder.
\end{abstract}

\section{B A C K G R O U N D}

\section{Description of the condition}

\section{Medical errors and the contribution of sleepiness}

Injuries to patients arising from medical errors are an important cause of avoidable mortality and morbidity, and are a great concern for clinicians, hospital managers and the public. In the UK, it is estimated that there are in excess of 850,000 incidents (constituting approximately $10 \%$ of admissions) which either harm or nearly harm a NHS patient every year, incurring $£ 2$ billion direct costs in additional hospital days alone (DoH 2000). In the USA, an estimated 44,000 to 98,000 deaths every year are caused by medical errors. Even when using the lowest estimate, this makes medical errors the eighth leading cause of death, exceeding the death toll from motor vehicle crashes, breast cancer and AIDS
(Kohn 1999). It is not just a concern for developed countries; in developing countries medical errors are likely to be more frequent than in the developed world, due to poor infrastructure, inadequate equipment, and other factors associated with severe underfinancing of health services (WHPA 2002).

There are numerous risk factors that can contribute to the occurrence of medical errors although the sleepiness of healthcare providers has been identified as being of concern (Gaba 2002; Jha 2001; Kohn 1999), and there is evidence of an association between medical errors and sleep deprivation (Eastridge 2003; Weinger 2002). Clinicians are required to have good attention, good judgment and quick reaction times, sometimes in high pressurised, emergency situations, all of which can be compromised by sleepiness (Jha 2001). The impairment of alertness associated with sleepiness, leads to a deterioration of performance, attention and motivation, diminishment of mental concentration and intellectual capacity (WHO 2004). A meta-analysis by Pilcher 1996, showed that the performance of sleep-deprived individuals was 
1.37 standard deviations lower than those with adequate sleep, and that partial sleep deprivation $(<5$ hours of sleep in a 24 hour period) had a greater negative effect on cognition than short or long term sleep deprivation (time without sleep $\leq 45$ hours and $>45$ hours respectively). Another meta-analysis by Philibert 2005, explored the effects of sleep deprivation on performance in resident physicians and non-physicians, finding that sleep deprivation of 24 to 30 hours reduced overall performance by one standard deviation and clinical performance by 1.5 standard deviations.

\section{Wider implications of sleepiness}

The harmful effects of errors caused by impaired alertness are not limited to the medical setting; they pose an important health risk in a number of sectors and industries, and their possible consequences vary enormously. A number of high profile human catastrophes and environmental disasters have been caused, at least in part, by human error attributed to sleepiness. These include the Three Mile Island nuclear plant incident, Chernobyl explosion (Mitler 1988) and the space shuttle Challenger explosion (Wilson 2005). Occupational injuries as a whole present an important public health issue and the effects of sleepiness are believed to be a significant risk factor. A number of observational studies have found that workers with higher levels of sleepiness have a greater risk of experiencing an occupational injury, than those with the lower levels of sleepiness (Melamed 2002; Swaen 2003).

In terms of transportation injuries, sleepiness is understood to be a major risk factor. A consensus statement endorsed by an international group of sleep experts states that "fatigue (sleepiness, tiredness) is the largest identifiable and preventable cause of accidents in transport operations (between 15\% and 20\% of all accidents), surpassing that of alcohol or drug related incidents in all modes of transportation. Official statistics often underestimate this contribution" (Akerstedt 2000). One survey conducted in England found that sleep-related incidents comprised $16 \%$ to $20 \%$ of all police-attended motor vehicle crashes (Horne 1995), and it is believed that relatively moderate levels of sleepiness impair performance to an extent that is equivalent to, or greater than, that currently acceptable for alcohol intoxication (Dawson 1997). Specifically in the field of aviation the reduction of aircraft crashes and incidents caused by human sleepiness is listed as one of the 'most wanted' transportation safety improvements by the USA's National Transportation Safety Board (NTSB 2008).

It should be noted that inadequate sleep is a risk factor for a number health problems. Total sleep deprivation is fatal in some animal species (Rechtschaffen 1989; WHO 2004) and short sleepers (< six hours) have a higher overall mortality rate (Wingard 1983). Insufficient sleep is also associated with an increased risk of obesity, type-2 diabetes, heart problems and dementia (Wilson 2005). Of particular interest to this systematic review is the diminished alertness and cognitive performance associated with sleep deprivation (Thomas 2000), including the adverse impact on vigilance
(Franzen 2008; Gillberg 1998), speech (Harrison 1997), decision making (Harrison 2000; Killgore 2006), divergent and flexible thinking (Harrison 1999; Horne 1988), and increased distractibility (Anderson 2006). Yet the extent of these negative impacts depends on other factors such as age, individual variability and other lifestyle factors such as alcohol consumption.

\section{Circadian rhythm sleep disorders}

Circadian rhythm sleep disorders (CRSDs) explain some of the sleepiness and associated impaired alertness that is prevalent in the population. The circadian rhythm describes the cyclical changes involving body temperature, hormone levels and sleep occurring over a 24 hour period, which are driven by our biological clock. This cycle is synchronised by rhythmic environmental cues, known as zeitgebers - the main zeitgebers are known to be the environmental light-dark cycle and the secretion of melatonin, both of which can be manipulated to induce a phase shift in an individual's circadian rhythm (Waterhouse 2007). The circadian rhythm dictates the times at which we feel the typical urge to sleep at night and wake in the morning. CRSDs occur when there is a misalignment between a person's sleep pattern and the sleep pattern desired (AASM 2001), which results in symptoms of sleepiness and insomnia.

Time zone change syndrome (i.e. jet lag) and shift work disorder (SWD) are two types of CRSD which can impair performance of workers in the health care and transportation industry, and are the focus of this review. These disorders can be considered distinct from the other CRSDs as sufferers of these conditions have circadian systems which function normally under usual circumstances (Sack 2007), which differs from the other disorders in which sufferers have an inherent problem with their circadian rhythm.

\section{Time zone change syndrome (jet lag)}

The American Academy of Sleep Medicine (AASM) describes jet lag as consisting "of varying degrees of difficulties in initiating or maintaining sleep, excessive sleepiness, decrements in subjective daytime alertness and performance, and somatic symptoms... following rapid travel across multiple timezones" (AASM 2001). Jet lag results from the misalignment between the circadian rhythm and the sleep-wake schedule in the new time zone. The severity of the symptoms of the disorder depend on the age of the traveller (severity increases with age), number of time zones crossed, direction of travel (eastward journeys are associated with more profound effects), time of travel and the individual's susceptibility (Waterhouse 2007). Symptoms alleviate as the body clock adjusts to new time zone, this adjustment takes a number of days, the exact number of which corresponds to approximately two-thirds of the number of time zones crossed (Waterhouse 2007). The detrimental effects of jet lag on alertness can have serious implications for those who do not have the opportunity to adjust to the new time zone, and 
are required to be sufficiently alert to perform tasks accurately and safely, such as aircrew and military personnel.

\section{Shift work disorder}

Shift work disorder (SWD) is described by the AASM as consisting "of symptoms of insomnia or excessive sleepiness that occur as transient phenomena in relation to work schedules". It mainly affects those persons whose working hours are scheduled during the habitual hours of sleep. The main complaint of sufferers is the inability to maintain a normal sleep duration when the major sleep episode is begun in the morning after a night shift. Workers engaged in early morning (starting between 04.00 and 07.00 ) or evening shift work, may also experience sleep difficulties associated with their working hours (AASM 2001). Excessive sleepiness often occurs during the shift work, thus impairing alertness. Over time some improvement in symptoms is observed, however, they tend to persist to some degree for the duration of the shift work, only alleviating once a regular daytime shift pattern is resumed (AASM 2001).

The number of people engaged in shift work has increased in response to the demands of a 24 hour global society. It is currently estimated that $15 \%$ to $20 \%$ of all workers are engaged in night or shift work in most industrialised countries (Bonneford 2004). A survey of workers in Detroit, USA, estimated that $10 \%$ of the night and rotating shift workers suffer from SWD (Drake 2004). Both the health care and transportation sectors employ a high proportion of shift workers in comparison to other industries (Beers 2000) as round the clock provision of services is required, thus it is likely that SWD is an important cause of impaired alertness in these individuals.

\section{Description of the intervention}

Adequate, quality natural sleep is by far the most effective and safe measure for preventing the detrimental effects of lack of sleep, including injury. However, there will always be a need for individuals to sleep and/or be alert at times which are different to those dictated by their circadian rhythm. Consequently there is a need for interventions which can safely alleviate the impairment of alertness associated jet lag and SWD. Effective interventions to prevent and treat the jet lag and SWD have the potential to reduce the number of errors resulting from impaired alertness, thus preventing the occurrence of injury to themselves, and to others, that may result.

Some interventions may aim to address the adverse effect on alertness arising from jet lag and SWD in two related, but distinct, ways. First, there are those interventions which aim to promote alertness during times of desired wakefulness (e.g. pharmacological stimulants) and those which aim to treat the insomnia symptom, and facilitate sleep during the desired times (e.g. pharmacological sedatives). Second, interventions may be employed to directly help the circadian rhythm to adjust to the new sleep-wake schedule (e.g. administration of bright light). A final type of intervention that might be employed are those which do not aim to treat an individual's symptoms, instead they attempt to directly prevent the occurrence of an error (e.g. alarm systems). In this systematic review we plan to review the evidence for the effects of all of the above types of interventions for use by those with jet lag or SWD.

\section{Why it is important to do this review}

As described above, the impaired alertness associated with the symptoms of jet lag and SWD is an important cause of injury and constitutes a significant threat to public safety.

The main focus of this review is on the prevention of medical errors and identification of effective interventions of relevance to the health care industry. However, the scope of the review will be broadened to include evidence from all sectors for a number of reasons. First, there is a dearth of quality intervention research data about ways to reduce medical errors and improve patient safety (Ioannidis 2001), yet other fields such as aviation and road safety have a more developed research tradition in prioritising public safety and risk management. There is, therefore, much potential for the development of strategies aimed at preventing medical errors to be informed by research from other industries. Second, the issue of impaired alertness resulting from jet lag and SWD is not a concern of just one sector, rather it has far-reaching implications. By reviewing all relevant evidence this review will be able to serve as a resource for all sectors, not solely health care, which seek to address the burden of injuries resulting from impaired alertness. This broad perspective also accounts for the inclusion of jet lag, which we recognise is unlikely to be an important cause of medical errors. However, we have chosen to include it as it is a potentially important cause of injury in other occupations such as the military and aviation. Furthermore, there are similarities between the two disorders in terms of aetiology (i.e. circadian desynchronisation) and symptoms (i.e. insomnia and sleepiness), thus we suggest that evidence regarding the effects of interventions for jet lag will have relevance to SWD, and vice versa.

To the best of our knowledge this will be the first systematic review and proposed meta-analysis aimed at assessing the effects of any intervention administered to sufferers of jet lag and SWD, on risk of injury. Although there is an existing Cochrane systematic review including trials examining the effects of melatonin for the prevention and treatment of jet lag (Herxheimer 2002), we do plan to include these studies in our review. We will however, have a primary focus on injury prevention and will be comparing the effects of melatonin with other intervention types in the context of both jet lag and SWD.

\section{O B J E C T IVES}


To assess the effects of interventions for preventing injuries caused by impaired alertness in persons with jet lag or shift work disorder.

\section{METHODS}

\section{Criteria for considering studies for this review}

\section{Types of studies}

- Randomised controlled trials.

- Randomised cross-over trials.

\section{Types of participants}

Persons of any age suffering from jet lag or engaged in shift work, and who are otherwise healthy.

Eligible shift workers may or may not have a formal diagnosis of SWD and could be engaged in early morning, late evening or night shift work.

The onset of jet lag or SWD may be real or induced (e.g. a simulated night shift in a laboratory setting).

\section{Types of interventions}

Any intervention aimed at preventing errors caused by alertness deficits associated with jet lag or shift work disorder.

Eligible interventions will incorporate:

- sedatives for facilitating sleep during episodes of insomnia;

- stimulants for promoting alertness during periods of desired wakefulness;

- interventions for facilitating adjustment of circadian rhythm through zeitgebers;

- interventions for preventing the occurrence of errors.

Anticipated interventions incorporate the following:

- Pharmaceutical stimulants (e.g. modafinil,

methylphenidate, pemoline);

- Pharmaceutical sedatives (e.g. melatonin, zolpidem);

- Napping strategies (nap frequency and duration);

- Shift/flight patterns;

- Dietary interventions (e.g. energy drinks, caffeinated beverages);

- Physical activity;

- Complementary and alternative therapies;

- Environmental interventions (e.g. light levels, ambient temperature, ventilation);

- Work schedules and hours;

- Sleep detection and warning equipment (e.g. wrist worn fatigue monitors such as the Fatigue Science's SleepBand ${ }^{\mathrm{TM}}$ );

- Education (e.g. sleep hygiene advice);
- Error prevention systems (e.g. alarms, computer systems).

\section{Types of outcome measures}

\section{Primary outcomes}

- Occurrence of injury.

- Occurrence of error (error as defined by the individual trial).

\section{Secondary outcomes}

- Tests of performance.

We anticipate that the included studies will use a variety of tests to measure cognitive function. We will therefore attempt to categorise the tests according to the type of cognitive construct under assessment. To do so we will refer to the classification described in Lezak 2004.

\section{Adverse events}

We also plan to collect data on the following potential adverse effects:

- Subsequent impairment of sleep architecture;

- Risk of dependence;

- Sleep inertia (the period of drowsiness and impaired performance which occurs immediately after waking).

\section{Search methods for identification of studies}

The searches will not be restricted by language or publication status.

\section{Electronic searches}

We will search the following electronic databases:

- CENTRAL;

- MEDLINE;

- EMBASE;

- PsycINFO;

- CINAHL

We will use the following MEDLINE strategy adapted as appropriate to the specifications of each database.

1. exp Aerospace Medicine/

2. exp Jet Lag Syndrome/

3. exp Sleep Disorders, Circadian Rhythm/

4. exp Work Schedule Tolerance/

5. (work* adj3 schedule* adj3 tolerance*).ab,ti.

6. ((sleep-wake or time-zone) adj3 (disorder* or syndrome*)).ab,ti. 
7. (circadian adj2 (dysrythmi* or disrupt* or disturb* or disorder*)).ab,ti.

8. (jet-lag* or jetlag* or shift-lag* or shiftlag).ab,ti.

9. ((shift* $\operatorname{adj}^{3}$ work $\left.^{*}\right)$ or shiftwork* or (night* adj3 shift*) or nightshift ${ }^{*}$ or $\left(\right.$ night $^{*}$ adj3 work* ${ }^{*}$ or nightwork* or (rotat* adj3 shift $\left.{ }^{*}\right)$ ).ab,ti.

10. $\exp$ Accidents, Aviation/pc [Prevention \& Control]

11. (aviation or airtravel or (air adj1 travel)).ti,ab.

12. or/1-11

13. randomi?ed.ab.

14. randomized controlled trial.pt.

15. controlled clinical trial.pt.

16. placebo.ab.

17. clinical trials as topic.sh.

18. randomly.ab.

19. trial.ti.

20. or/13-19

21. humans.sh.

22. 20 and 21

23. 12 and 22

\section{Searching other resources}

We will search the following Internet resources:

- Computer Retrieval of Information on Scientific Projects;

- Current Controlled Trials;

- Defense Technical Information Center;

- Latin American and Caribbean Health Sciences Literature;

- Monash Accident Research Centre (www.monash.edu.au/ muarc);

- NASA Technical Reports Server;

- National Highway Traffic Safety Administration;

- National Institute for Occupational Safety and Health (www.cdc.gov/niosh);

- National Technical Information Service;

- National Transportation Library Integrated Search;

- Campbell Collaboration Social, Psychological, Educational \& Criminological Trials Register;

- Swedish National Road and Transport Research Institute (VTI);

- SWOV - Institute for Road Safety Research;

- Transportation Research Board;

- Transportation Research Library;

- Zetoc.

These resources will be searched using keywords selected from the above MEDLINE strategy.

We will also screen the electronic abstracts/proceedings of the following meetings:

- European Sleep Research Society;

- International Sleep Disorders Forum;

- World Congress of the World Association of Sleep Medicine.
In addition, we will search the reference lists of the eligible studies and any review articles for further potentially eligible articles. We will contact experts in the field as well as search the Internet using the Google (www.google.com) search engine with selected terms from the above strategy, for any further unpublished or grey literature.

\section{Data collection and analysis}

\section{Selection of studies}

Two authors will independently examine titles, abstracts, and keywords of electronic records for eligibility. We will obtain the full text of all potentially relevant reports of trials and two authors will independently assess whether each meet the pre-defined inclusion criteria. We will resolve any disagreement through discussion.

\section{Data extraction and management}

Two authors will extract data from each eligible study using a standard form that we will develop specifically for this review. We will extract data on the following:

- study design;

- participant characteristics;

- intervention characteristics;

- outcome measures;

- statistical analysis.

\section{Assessment of risk of bias in included studies}

The risk of bias in included studies will be assessed using the Cochrane Collaboration's recommended tool described in Higgins 2008. This tool assesses the following six domains:

- Sequence generation (was the allocation sequence adequately generated?);

- Allocation concealment (was allocation adequately concealed?);

- Blinding (was knowledge of the allocated intervention adequately prevented during the study?);

- Incomplete outcome data (were incomplete outcome data adequately addressed?);

- Selective outcome reporting (are reports of the study free of suggestion of selective outcome reporting?);

- Other issues (was the study apparently free of other problems that could put it at a high risk of bias?).

Risk of bias tables based on the above criteria will be completed. These will incorporate the review authors' judgement ('Yes' for low risk of bias; 'No' for high risk of bias, or 'Unclear') and description of the design, conduct or observations that underlie the judgement, for each domain in each included study. 


\section{Measures of treatment effect}

We anticipate that trial results will be presented using a combination of dichotomous and continuous data.

For dichotomous outcomes we plan to calculate relative risks (RR) and $95 \%$ confidence intervals $(\mathrm{CIs})$ for each trial.

For continuous data the weighted mean difference (WMD) and 95\% CIs will be calculated when the same scale is used in a similar manner across studies. If results for continuous outcomes are reported using different scales or different versions of the same scale, we will calculate the standardised mean difference (SMD) and $95 \%$ CIs.

\section{Unit of analysis issues}

It is anticipated that we will identify a number of randomised cross-over studies which will be eligible for inclusion. If there are appropriate data we will incorporate data from all experimental periods into the analyses according to the methods described in Elbourne 2002.

\section{Dealing with missing data}

We will attempt to contact the original investigators to obtain any missing data that are required.

\section{Assessment of heterogeneity}

We will examine trial characteristics in term of participants, interventions and outcomes for evidence of clinical heterogeneity. Statistical heterogeneity will be examined by both the I-squared and chi-squared tests. The I-squared test describes the percentage of total variation across studies due to heterogeneity rather than chance. A value of $0 \%$ indicates no observed heterogeneity, and larger values show increasing heterogeneity; substantial heterogeneity is considered to exist when I-squared $>50 \%$. For the chi-squared statistic, a $P$ value of $<0.10$ will be used to indicate the presence of statistically significant heterogeneity.

Possible sources of heterogeneity will be assessed by subgroup analyses as described below.

\section{Assessment of reporting biases}

We will investigate the presence of reporting bias using funnel plots.

\section{Data synthesis}

If we judge that the trials are clinically and statistically homogenous, we will pool the data using the fixed-effects model.

\section{Subgroup analysis and investigation of heterogeneity}

If there are sufficient data we plan to perform the following subgroup analyses:

- Age - persons aged $>60$ years old versus persons aged 18 to 60 years versus children aged $<18$ years;

- Environment - simulated, transportation, clinical, military;

- Allocation concealment - adequate versus inadequate.

\section{Sensitivity analysis}

If there are appropriate data we will conduct the following sensitivity analyses:

- meta-analysis - comparison of fixed-effect versus randomeffects methods.

\section{ACKNOW LEDGEMENTS}

Thanks to the peer referees for their valuable comments.

\section{RE F E R E NCES}

\section{Additional references}

\section{AASM 2001}

American Academy of Sleep Medicine. The International

Classification of Sleep Disorders, revised. http://

www.absm.org/PDF/ICSD.pdf 2001.

\section{Akerstedt 2000}

Akerstedt T. Consensus statement: fatigue and accidents in transports operations. Journal of Sleep Research 2000;9:395.

Anderson 2006

Anderson C, Horne JA. Sleepiness enhances distraction during a monotonous task. Sleep 2006;29(4):573-6. [PUBMED: 16676792]

\section{Beers 2000}

Beers TM. Flexible schedules and shift work: replacing the '9-to-5' workday?. Monthly Labor Review 2000; June:33-40.

Bonneford 2004

Bonneford A, Tassi P, Roge J, Muzet A. A critical review of techniques aiming at enhancing and sustaining worker's alertness during the night shift. Industrial Health 2004;42 (1):1-14.

Dawson 1997

Dawson D, Reid K. Fatigue, alcohol and performance impairment. Nature 1997;388(6639):235.

DoH 2000

Department of Health. An Organisation with a Memory. 
Report of an expert group on learning from adverse events in the NHS chaired by the Chief Medical Officer. 2000.

\section{Drake 2004}

Drake CL, Roehrs T, Richardson G, Walsh JK, Roth T. Shoft work sleep disorder: prevalence and consequences beyond that of symptomatic day workers. Sleep 2004;27(8): 1453-62.

Eastridge 2003

Eastridge BJ, Hamilton EC, O'Keefe GE, Rege RV, Valentine RJ, Jones DJ, et al.Effect of sleep deprivation on the performance of simulated laparoscopic surgical skill. American Journal of Surgery 2003;186(2):169-74.

Elbourne 2002

Elbourne DR, Altman DG, Higgins JPT, Curtain F, Worthington HV, Vail A. Meta-analysis involving crossover trials: methodological issues. International Journal of Epidemiology 2002;31:140-9.

\section{Franzen 2008}

Franzen PL, Siegle GJ, Buysse DJ. Relationships between affect, vigilance, and sleepiness following sleep deprivation. Journal of sleep research 2008;17(1):34-41. [PUBMED: 18275553]

Gaba 2002

Gaba DM, Howard SK. Patient safety: fatigue among clinicians and the safety of patients. New England Journal of Medicine 2002;347(16):1249-55.

Gillberg 1998

Gillberg M, Akerstedt T. Sleep loss and performance: no "safe" duration of a monotonous task. Physiology \& behavior 1998;64(5):599-604. [PUBMED: 9817569]

Harrison 1997

Harrison Y, Horne JA. Sleep deprivation affects speech. Sleep 1997;20(10):871-7. [PUBMED: 9415947]

\section{Harrison 1999}

Harrison Y, Horne JA. One Night of Sleep Loss Impairs Innovative Thinking and Flexible Decision Making. Organizational behavior and human decision processes 1999; 78(2):128-45. [PUBMED: 10329298]

\section{Harrison 2000}

Harrison Y, Horne JA. The impact of sleep deprivation on decision making: a review. Journal of experimental psychology. Applied 2000;6(3):236-49. [PUBMED: 11014055]

Herxheimer 2002

Herxheimer A, Petrie KJ. Melatonin for the prevention and treatment of jet lag. Cochrane Database of Systematic Reviews 2002, Issue 2. [DOI: 10.1002/14651858.CD001520]

Higgins 2008

Higgins JPT, Green S (editors). Cochrane Handbook for Systematic Reviews of Interventions Version 5.0.0 [updated February 2008]. The Cochrane Collaboration, 2008. Available from www.cochrane-handbook.org. 2008.

Horne 1988

Horne JA. Sleep loss and "divergent" thinking ability. Sleep 1988;11(6):528-36. [PUBMED: 3238256]
Horne 1995

Horne JA, Reyner LA. Sleep related vehicle accidents. $B M J$ 1995;310(6979):565-7.

\section{Ioannidis 2001}

Ioannidis JPA, Lau J. Evidence on interventions to reduce medical errors. An overview and recommendation for future research. Journal of General Internal Medicine 2001; 16:325-34.

\section{Jha 2001}

Jha AK, Duncan BW, Bates DW. Fatigue, sleepiness, and medical errors. Making health care safer. A critical analysis of patient safety practices 2001; Vol. Chapter 46.

Killgore 2006

Killgore WD, Balkin TJ, Wesensten NJ. Impaired decision making following $49 \mathrm{~h}$ of sleep deprivation. Journal of sleep research 2006;15(1):7-13. [PUBMED: 16489997]

Kohn 1999

Kohn LT, Corrigan JM, Donaldson MS, editors. To err is human. Building a safer health system. Institute of Medicine, National Academy Press, USA 1999.

\section{Lezak 2004}

Lezak MD, Howieson DB, Loring DW. Neuropsychological Assessment. Fourth. New York: Oxford University Press, 2004.

\section{Melamed 2002}

Melamed S, Okenberg A. Excessive daytime sleepiness and risk of occupational injuries in non-shift daytime workers. Sleep 2002;25(3):315-22.

Mitler 1988

Mitler MM, Carskadon MA, Czeisler CA, Dement WC, Dinges DF, Graeber RC. Catastrophes, sleep, and public policy: consensus report. Sleep 1988;11(1):100-9.

\section{NTSB 2008}

National Transportation Safety Board. NTSB most wanted list. Transportation safety improvements. http:/ /www.ntsb.gov/Recs/brochures/MostWanted_2008.pdf 2008.

\section{Philibert 2005}

Philibert I. Sleep loss and performance in residents and nonphysicians: a meta-analytic examination. Sleep 2005;28 (11):1392-1401.

\section{Pilcher 1996}

Pilcher JJ, Huffcutt AI. Effects of sleep deprivation on performance: a meta-analysis. Sleep 1996;19(4):318-26.

\section{Rechtschaffen 1989}

Rechtschaffen A, Bergmann BM, Everson CA, Kushida CA, Gilliland MA. Sleep deprivation in the rat: X. Integration and discussion of the findings. Sleep 1989;12(1):68-87.

\section{Sack 2007}

Sack RL, Auckley D, Auger RR, Carskadon MA, Wright KP, Vitiello MV, et al.Circadian rhythm sleep disorders: part I, basic principles, shift work and jet lag disorders. An American Academy of Sleep Medicine review. Sleep 2007; 30(11):1460-83. 
Swaen 2003

Swaen GM, Van Amelsvoort LG, Bultmann U, Kant IJ.

Fatigue as a risk factor for being injured in an occupational accident: results from the Maastricht Cohort Study. Occupational \& Environmental Medicine 2003;60(suppl 1): i88-92.

Thomas 2000

Thomas M, Sing H, Belenky G, Holcomb H, Mayberg $\mathrm{H}$, Dannals R, et al.Neural basis of alertness and cognitive performance impairments during sleepiness. I. Effects of $24 \mathrm{~h}$ of sleep deprivation on waking human regional brain activity. Journal of Sleep Research 2000;9:335-52.

Waterhouse 2007

Waterhouse J, Reilly T, Atkinson G, Edwards B. Jet lag: trends and coping strategies. Lancet 2007;369(9567): 1117-29.
Weinger 2002

Weinger MB, Ancoli-Israel S. Sleep deprivation and clinical performance. JAMA 2002;287(8):955-7.

WHO 2004

World Health Organization. WHO technical meeting on sleep and health. World Health Organization Regional Office for Europe 2004.

\section{WHPA 2002}

World Health Professions Alliance. Patient Safety factsheet. www.whpa.org/factptsafety.htm accessed 24/7/ 07.

\section{Wilson 2005}

Wilson JF. Is sleep the new vital sign?. Annuals of Internal Medicine 2005;142(10):877-80.

\section{Wingard 1983}

Wingard DL, Berkman LF. Mortality risk associated with sleeping patterns among adults. Sleep 1983;6(2):102-7.

* Indicates the major publication for the study

\section{H I S T O R Y}

Protocol first published: Issue 2, 2009

\section{CONTRIBUTIONS OFAUTHORS}

KK wrote the protocol.

PJE and IR helped conceptualise the review and commented on the draft protocol.

KB helped to devise the search strategy and commented on the draft protocol.

LF commented on the draft protocol.

\section{DECLARATIONSOF INTEREST}

None known.

\section{SOURCES OF SUPPORT}

\section{Internal sources}

- No sources of support supplied 


\section{External sources}

- National Institute for Health Research, UK.

NHS Cochrane Collaboration Programme Grant Scheme 